Alumina nanoparticles find an application to reduce the ionic effects of ferroelectric liquid crystal

This article has been downloaded from IOPscience. Please scroll down to see the full text article.

2011 J. Phys. D: Appl. Phys. 44315404

(http://iopscience.iop.org/0022-3727/44/31/315404)

View the table of contents for this issue, or go to the journal homepage for more

Download details:

IP Address: 14.139.60.97

The article was downloaded on 17/08/2011 at 06:34

Please note that terms and conditions apply. 


\title{
Alumina nanoparticles find an application to reduce the ionic effects of ferroelectric liquid crystal
}

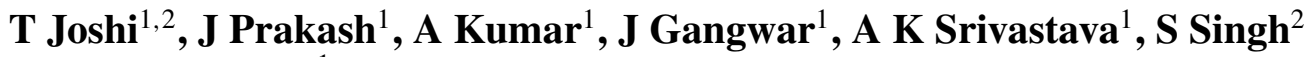 \\ and $\mathrm{A} M$ Biradar $^{1}$ \\ ${ }^{1}$ National Physical Laboratory, Council of Scientific and Industrial Research, Dr K. S. Krishnan Road, \\ New Delhi-110012, India \\ ${ }^{2}$ Department of Physics, Banaras Hindu University, Varanasi- 221 005, India \\ E-mail: abiradar@mail.nplindia.ernet.in
}

Received 26 March 2011, in final form 22 June 2011

Published 15 July 2011

Online at stacks.iop.org/JPhysD/44/315404

\begin{abstract}
We observed that the doping of alumina nanoparticles (AL-NPs) has suppressed the undesired ionic effect in ferroelectric liquid crystals (FLCs). The pure and AL-NPs doped FLC cells were analysed by means of dielectric spectroscopy and electrical resistivity/conductivity measurements. Dielectric loss spectra confirmed the disappearance of the low-frequency relaxation peak, which appears due to the presence of ionic impurities in the FLC materials. The reduction of ionic effects has been attributed to the strong adsorption of ionic impurities on the surface of AL-NPs. The adsorption capability of AL-NPs has been studied with both the size and their concentration in FLC material. This study would be helpful to minimize the undesired ionic effects of LC-based display devices.
\end{abstract}

(Some figures in this article are in colour only in the electronic version)

Display devices based on liquid crystal (LC) materials have been in great demand over the last few decades due to their advantages over other display devices. The LC materials have been employed in various switchable electro-optic devices due to their essential anisotropic (optical and dielectric) behaviour. The majority of the display and non-display devices have been based on nematic LCs. Recently, various interesting electrooptical properties such as good optical contrast, low threshold voltage, memory effect, fast response of ferroelectric liquid crystals (FLCs) have attracted a great deal of attention from the researchers in the world. The incorporation of nanomaterials such as carbon nanotubes, zinc oxide nanoparticles and gold nanoparticles in FLCs have further improved their electrooptical properties in the form of faster response [1], low threshold [2] and non-volatile memory [3]. However, the presence of undesired ionic impurities in LC materials is still a major challenge to be taken care of as these impurities can cause the degradation of the display devices based on these materials [4-6]. These impurities enter within LCs either through the atmosphere or alignment layer or during their synthesis. Ions within LCs generate electric field which could lead to polar surface interactions and thus anchoring energy and driving voltage could be changed $[7,8]$. These ions may cause grey-level shift, image sticking and slow response time of the LC device [9]. Some remarkable contributions have been made in the direction of minimization of ionic impurities by doping insulating nanoparticles in nematic LCs. The authors observed a remarkable reduction in the transient current and threshold voltage of nematic LC doped with diamond powder $[10,11]$. The same effects were observed by doping $\mathrm{ZnO}$, $\mathrm{TiO}_{2}$ and $\mathrm{Si}_{3} \mathrm{~N}_{4}$ nanoparticles in nematic LCs by Chen et al [12]. Lee et al [13] observed that a degraded nematic LC material could be recovered by adding complex coordination polymers via chemical adsorption. Liu et al [14] showed that inorganic montmorillonite (MMT) nano-platelets were able to suppress the ionic effect by trapping the ions. Onishi et al [15] developed a FLC display device with good display quality without the deterioration of display characteristics, as a result of trapping the ionic impurities effectively by adding an organic material which traps ions and lowers conductivity of a LC layer. All these studies were focused on the fact of increasing ion contamination in $\mathrm{LC}$ as time passes, e.g. permeation of 
atmospheric water into LC preserved in sealed containers, injection of ions from alignment layers, sealants and LC ageing $[16,17]$.

In this paper, the suppression of ionic effects of FLC materials doped with alumina nanoparticles (AL-NPs) has been presented. The effect of AL-NPs doping has been analysed by means of dielectric spectroscopy and resistivity/conductivity measurements. We found that the defect sites existing over the surfaces of AL-NPs have adsorbed the undesired ionic impurities present in FLCs. Dielectric loss spectra of AL-NPs doped FLC cells clearly reflected the suppression of ionic effects in the form of disappearance of low-frequency relaxation, generally caused by ions.

To synthesize AL-NPs, saturated solution of aluminium nitrate was made by dissolving it in distilled water with continuous stirring until no more aluminium nitrate is soluble in it. Thus clear sols were obtained. The sols thus obtained were heated at $100{ }^{\circ} \mathrm{C}$ for $30 \mathrm{~min}$, so that a gel is formed and then annealed at different temperatures ranging from 800 to $1200{ }^{\circ} \mathrm{C}$ for $2 \mathrm{~h}$ in a furnace to get AL-NPs of different sizes (4-50 nm). High-resolution transmission electron microscopy (HRTEM model Tecnai G2 F30 STWIN with FEG source, operated at the electron accelerating voltage of $300 \mathrm{kV}$ ) and $\mathrm{x}-$ ray diffraction (XRD model Bruker AXS D8 Advance X-ray Diffractometer, $\mathrm{Cu}-\mathrm{K} \alpha$ wavelength, $\lambda=1.54059 \AA$ Á, scanning $2 \theta$ range from $20^{\circ}$ to $80^{\circ}$ ) were performed to characterize the prepared AL-NPs. The suitable amount of nanopowder of ALNPs was first mixed with the FLC materials. The mixture was then ultrasonicated for about $40 \mathrm{~min}$ in the isotropic phase of the respective material to ensure the homogeneous dispersion of AL-NPs in FLC material.The pure and AL-NPs (of different concentrations and sizes) doped KCFLC 7S mixtures were then introduced into the LC sample cells by means of capillary action at temperatures just above the isotropic transition temperature. We used the sample cells consisting of conducting $(\sim 30 \Omega / \square)$ indium tin oxide (ITO) coated glass plates. The ITO patterns used as electrodes were prepared by the photolithographic technique. Homogeneously aligned cells were prepared using the rubbed polyimide technique. The thickness between the two plates was maintained using Mylar spacers. The dielectric and resistivity/conductivity studies of the AL-NPs/FLC mixtures have been performed using an impedance analyser $6540 \mathrm{~A}$ (Wayne Kerr, U. K). The phase sequences of FLC mixtures used are as follows:

cryt $\stackrel{?}{\longleftrightarrow} \mathrm{SmC}^{*} \stackrel{73^{\circ} \mathrm{C}}{\longleftrightarrow} \mathrm{SmA} \stackrel{100^{\circ} \mathrm{C}}{\longleftrightarrow} \mathrm{N} \stackrel{114.5^{\circ} \mathrm{C}}{\longleftrightarrow}$ iso $\mathrm{KCFLC} 7 \mathrm{~S}$ cryt $\stackrel{?}{\longleftrightarrow} \mathrm{SmC}^{*} \stackrel{64.5^{\circ} \mathrm{C}}{\longleftrightarrow} \mathrm{SmA} \stackrel{99.5^{\circ} \mathrm{C}}{\longleftrightarrow} \mathrm{N} \stackrel{112^{\circ} \mathrm{C}}{\longleftrightarrow}$ iso. KCFLC $10 \mathrm{~S}$

Figure 1 shows the HRTEM micrograph of AL-NPs obtained by annealing the gel at $1000^{\circ} \mathrm{C}$ for $2 \mathrm{~h}$ in the furnace. The results obtained using HRTEM elucidated the presence of ultra-fine scaled nanoparticles of size between 20 and $30 \mathrm{~nm}$ with the crystallographic phase of hexagonal $\alpha-\mathrm{Al}_{2} \mathrm{O}_{3}$ having lattice constants $a=0.475 \mathrm{~nm}, c=1.299 \mathrm{~nm}$ and $\gamma=120^{\circ}$. The inset in figure 1 reveals further the stacking of $0 \overline{2} \overline{2} 0$ planes with the interplanar spacing of about $0.26 \mathrm{~nm}$ of a nanocrystallite of size approximately $25 \mathrm{~nm}$.

The effect of AL-NPs of various sizes on the alignment of FLCs was analysed by observing the optical micrographs

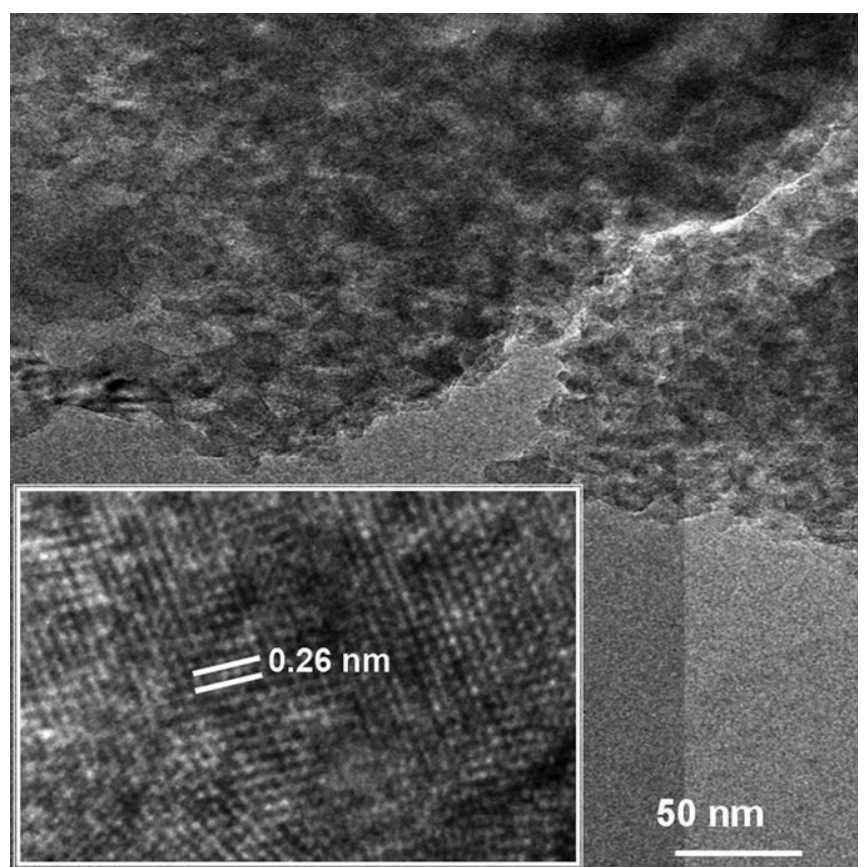

Figure 1. HRTEM micrograph showing the size distribution of AL-NPs. Inset shows lattice scale image of nano-crystallites.
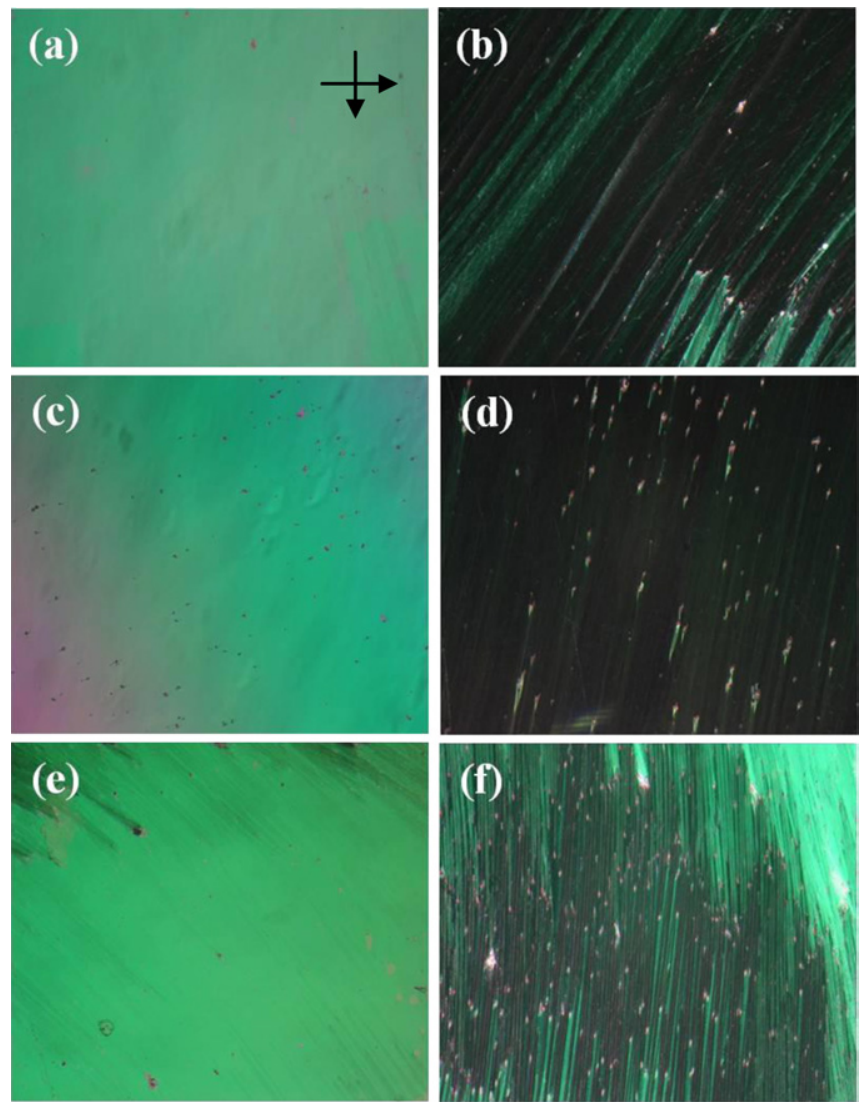

Figure 2. Polarizing micrographs showing the bright and dark states of $1 \mathrm{wt} \%$ AL-NPs of different sizes $(a),(b)$ for $4-7 \mathrm{~nm} ;(c),(d)$ for 20-30 nm and $(e),(f)$ for 30-50 nm doped KCFLC 7S material at room temperature (RT). Crossed arrows show the crossed polarizers and are kept crossed for all samples. 

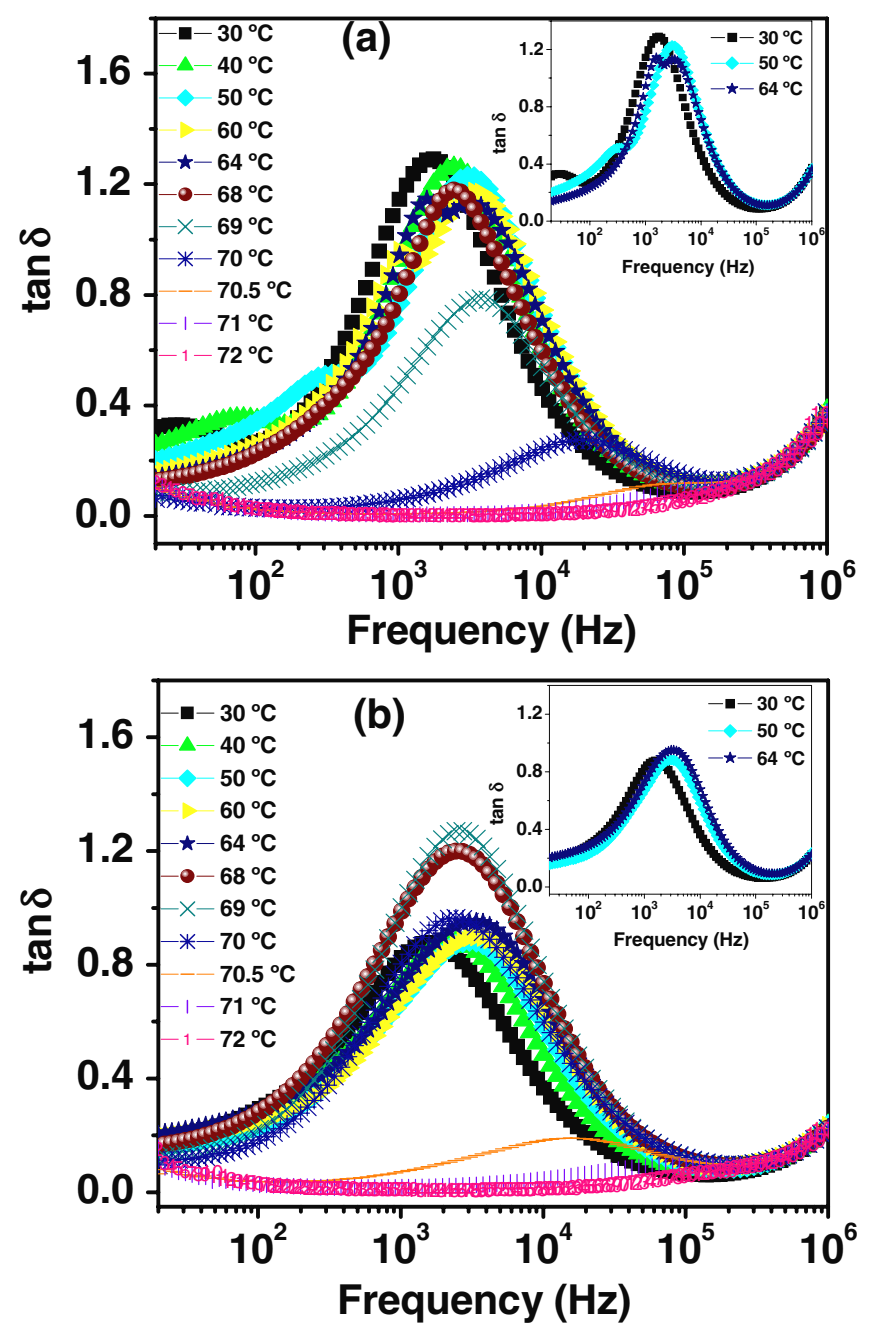

Figure 3. Behaviour of dielectric loss factor $(\tan \delta$ ) with frequency for homogeneously aligned $4.2 \mu \mathrm{m}$ thick cells of $(a)$ pure and $(b)$ $1 \mathrm{wt} \%$ AL-NPs of size $20-30 \mathrm{~nm}$ doped KCFLC $7 \mathrm{~S}$ material. Inset highlighting same curves for some temperatures.

of the pure and AL-NPs doped KCFLC 7S cells. Figure 2 shows the optical micrographs of the bright and dark states of $1 \mathrm{wt} \%$ AL-NPs (of different sizes) doped KCFLC 7S material. The presence of AL-NPs in the FLC material can be clearly seen from the micrographs of dark states in the form of light scattering centres (figures $2(b),(d)$ and $(f)$ ). We found that the doping of $1 \mathrm{wt} \%$ of AL-NPs in the size range 4-30 $\mathrm{nm}$ did not perturb the alignment of FLC material much. However, on increasing the size $(>30 \mathrm{~nm}$ ) of AL-NPs, the alignment of FLC material was degraded which can be seen in the form of large aggregates (figures $2(e)$ and $(f)$ ). As AL-NPs in the size range 20-30 nm did not perturb the FLC alignment remarkably, we carried out further studies on KCFLC 7S/AL-NPs composites by doping $1 \mathrm{wt} \%$ AL-NPs of size $20-30 \mathrm{~nm}$. The effect of ALNPs on the dielectric relaxation processes was analysed by observing the behaviour of pure and AL-NPs doped KCFLC $7 \mathrm{~S}$ cells. Figure 3 shows the behaviour of dielectric loss factor $(\tan \delta)$ with frequency at different temperatures of $4.2 \mu \mathrm{m}$ thick homogeneously aligned cells of pure (figure 3(a)) and $1 \mathrm{wt} \%$ AL-NPs doped (figure 3(b)) KCFLC 7S material. As can be seen from figure $3(a)$, a low-frequency $(<100 \mathrm{~Hz})$
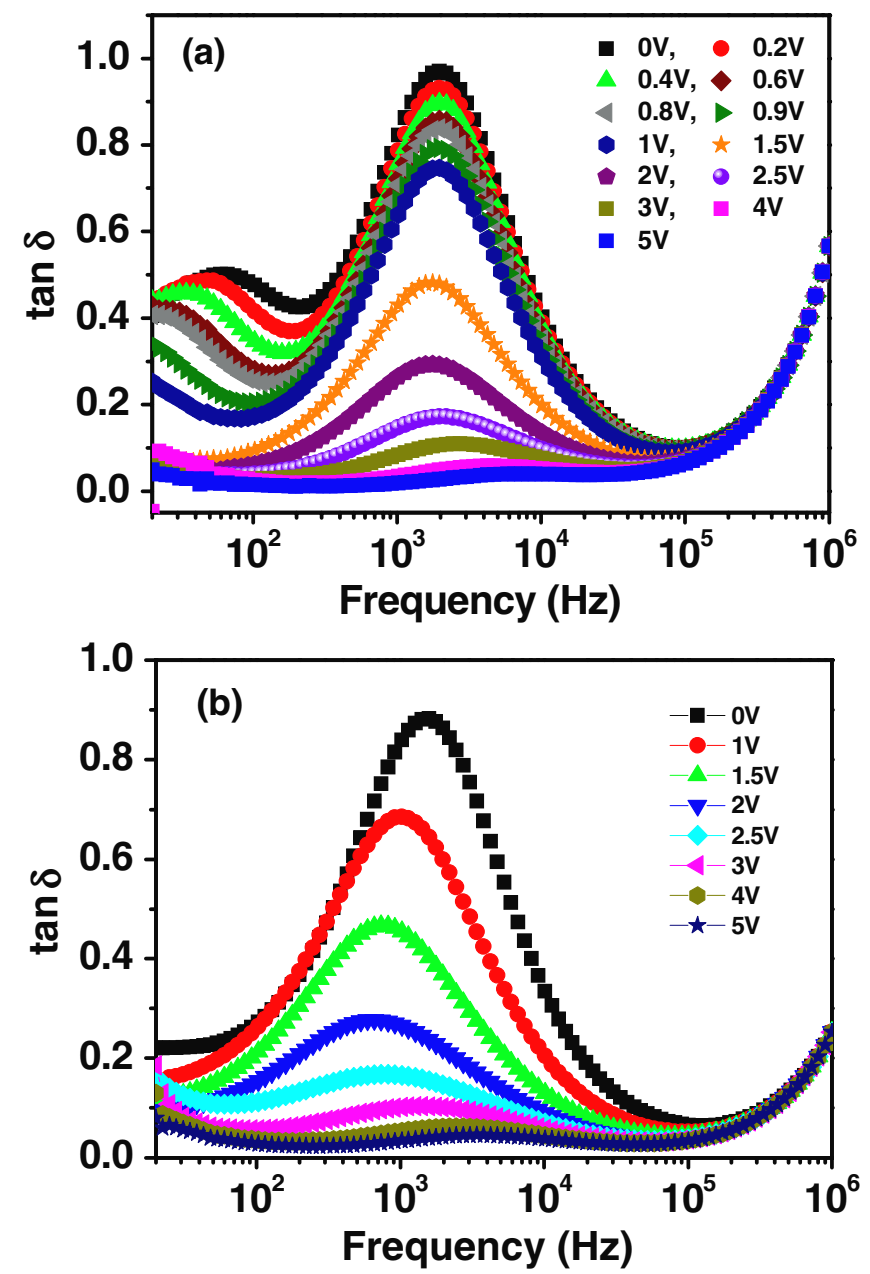

Figure 4. Behaviour of low-frequency relaxation peak of $(a)$ pure and (b) $1 \mathrm{wt} \%$ AL-NPs of size 20-30 nm doped KCFLC 7S material on the application of different dc biases at RT.

relaxation mode along with the usual Goldstone mode (GM) is observed in the case of pure KCFLC $7 \mathrm{~S}$ material. The collective and non-collective dielectric processes have usually been observed in the form of different relaxation modes in the smectic $\mathrm{C}^{*}\left(\mathrm{SmC}^{*}\right)$ phase of FLC materials. The main collective processes involve GM, connected with the phase fluctuations in the azimuthal orientation of the director and soft mode due to fluctuations in the amplitude of the tilt angle [18-20]. The appearance of the low-frequency mode in KCFLC 7S material has been studied earlier and was attributed to the ionization-recombination assisted diffusion of slow ions in planar alignment configuration [21]. The presence of ionic impurities within the FLC material is visible as a distinct peak at room temperature which shifted to higher frequencies on increasing the temperature and ultimately merges with GM near $\mathrm{SmC}^{*}-\mathrm{SmA} *$ transition. In figure $3(b)$, it can be seen that the low-frequency mode has disappeared by doping AL-NPs into KCFLC $7 \mathrm{~S}$ material. The disappearance of the lowfrequency mode suggested the adsorption of ions responsible for the generation of low-frequency relaxation. Moreover, the low-frequency peak did not appear anywhere on increasing the temperature to higher values. The presence or the absence of low-frequency peak can be clearly visualized in the insets of 

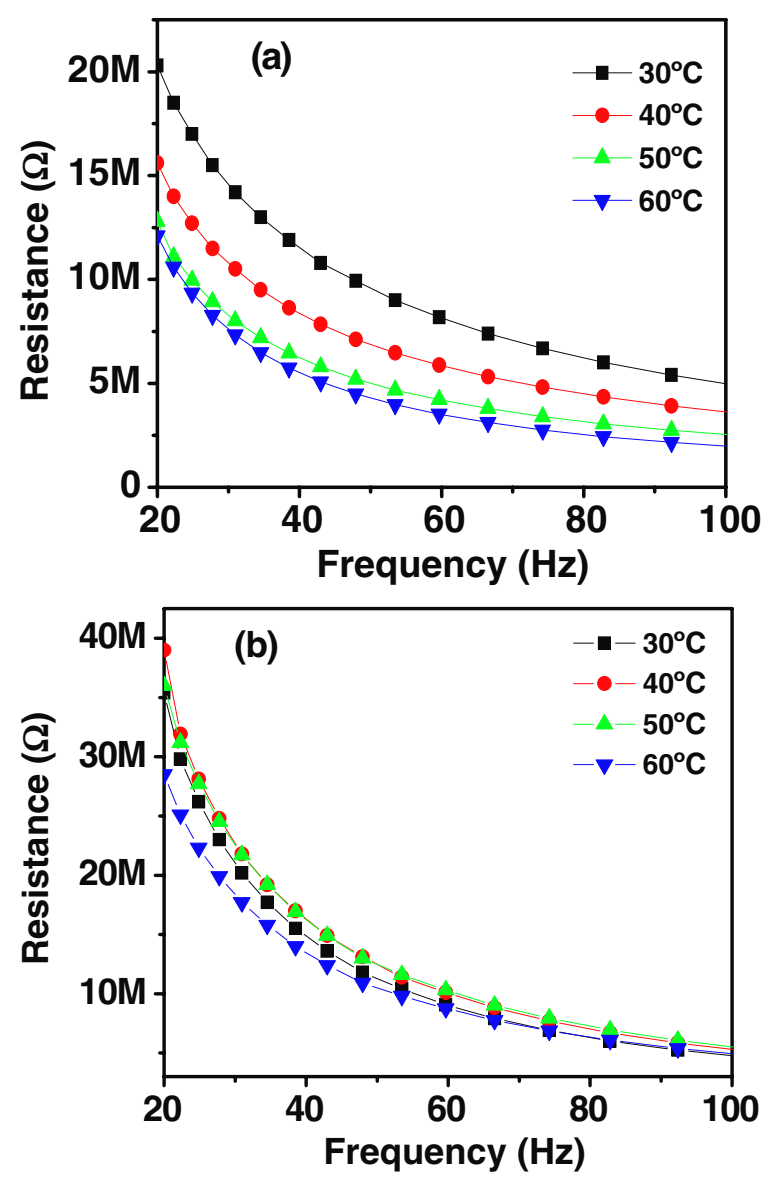

Figure 5. Behaviour of electrical resistance $(R)$ of $(a)$ pure and $(b)$ $1 \mathrm{wt} \%$ AL-NPs of size 20-30 nm doped KCFLC 7S material with frequency for homogeneously aligned $4.2 \mu \mathrm{m}$ thick cells.

figure 3 in which the variation of $\tan \delta$ with frequency at some selected temperatures is highlighted. The effect of doping concentration of AL-NPs on their adsorption capability has been observed by varying the concentration of 20-30 nm ALNPs into KCFLC 7S material from 0.5 to $3 \mathrm{wt} \%$. We found that $1 \mathrm{wt} \%$ AL-NPs with $20-30 \mathrm{~nm}$ size range are suitable to effectively suppress the ionic contributions of FLC material. It is noticeable that the presence of AL-NPs did not affect the behaviour of $\mathrm{GM}$ and $\mathrm{SmC}^{*}-\mathrm{SmA} *$ transition temperature of KCFLC 7S material. The effect of dc bias field on the lowfrequency mode was also observed by applying different $\mathrm{dc}$ biases across the cells (figure 4). The low-frequency peak has been shifted towards the low-frequency side by applying dc bias. The shifting of the low-frequency peak towards the low-frequency side has already been explained by us [21]. The suppression of ionic contributions of KCFLC 7S material has further been confirmed by observing the behaviour of electrical resistance $(R)$ of pure and AL-NPs doped KCFLC $7 \mathrm{~S}$ material. Figure 5 shows the behaviour of $R$ of pure and $1 \mathrm{wt} \%$ AL-NPs doped KCFLC 7S material with frequency at different temperatures. In the case of pure KCFLC 7S material, the value of $R$ was observed around $20 \mathrm{M} \Omega$ at $20 \mathrm{~Hz}$ frequency, which was decreased on increasing the temperature to higher values (figure 5(a)). Figure $5(b)$ shows the variation of $R$ with frequency at different temperatures for $1 \mathrm{wt} \%$ AL-NPs doped KCFLC 7S material. The resistance of $1 \mathrm{wt} \%$ AL-NPs
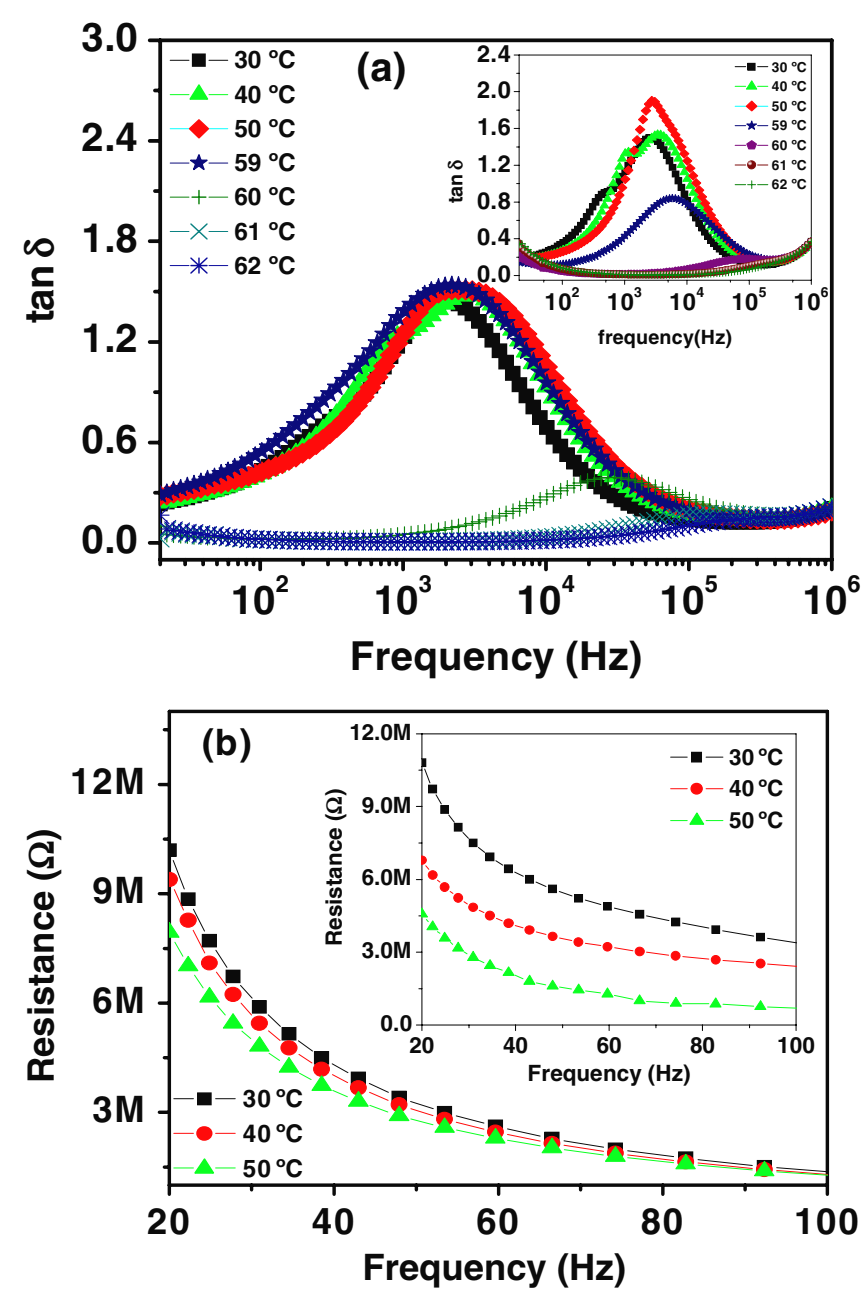

Figure 6. Behaviour of $\tan \delta$ versus frequency $(a)$ and electrical resistance $(b)$ for $1 \mathrm{wt} \%$ AL-NPs of size 20-30 nm doped KCFLC $10 \mathrm{~S}$ in homogeneously aligned $4.3 \mu \mathrm{m}$ thick cells and comparison with pure cells having same configuration (inset).

doped KCFLC 7S material was observed around $35 \mathrm{M} \Omega$ at $20 \mathrm{~Hz}$ frequency at room temperature, which is higher than that of the pure one. The increased value of resistance in the case of AL-NPs doped KCFLC 7S material implied the reduction of ionic impurities due to doping of the AL-NPs. Moreover, the value of resistance did not change remarkably on increasing the temperature to higher values in the case of AL-NPs doped KCFLC 7S material. This suggests that the ion adsorption capability of AL-NPs is almost temperature independent in nature.

We have also observed the effect of AL-NPs on another FLC material, namely KCFLC 10S, which is of the same series from which KCFLC 7S was. Figure 6 shows the behaviour of $\tan \delta$ with frequency for pure and $1 \mathrm{wt} \%$ AL-NPs doped KCFLC $10 \mathrm{~S}$ material at different temperatures. The occurrence of low-frequency relaxation in the case of pure KCFLC 10S material due to the presence of ionic impurities can be easily seen from the inset of figure $6(a)$. The higher values of low-frequency losses suggest the presence of more ionic impurities. The doping of AL-NPs suppressed the ionic effect of KCFLC 10S material as the low-frequency mode associated with ionic effects has disappeared in the case of 

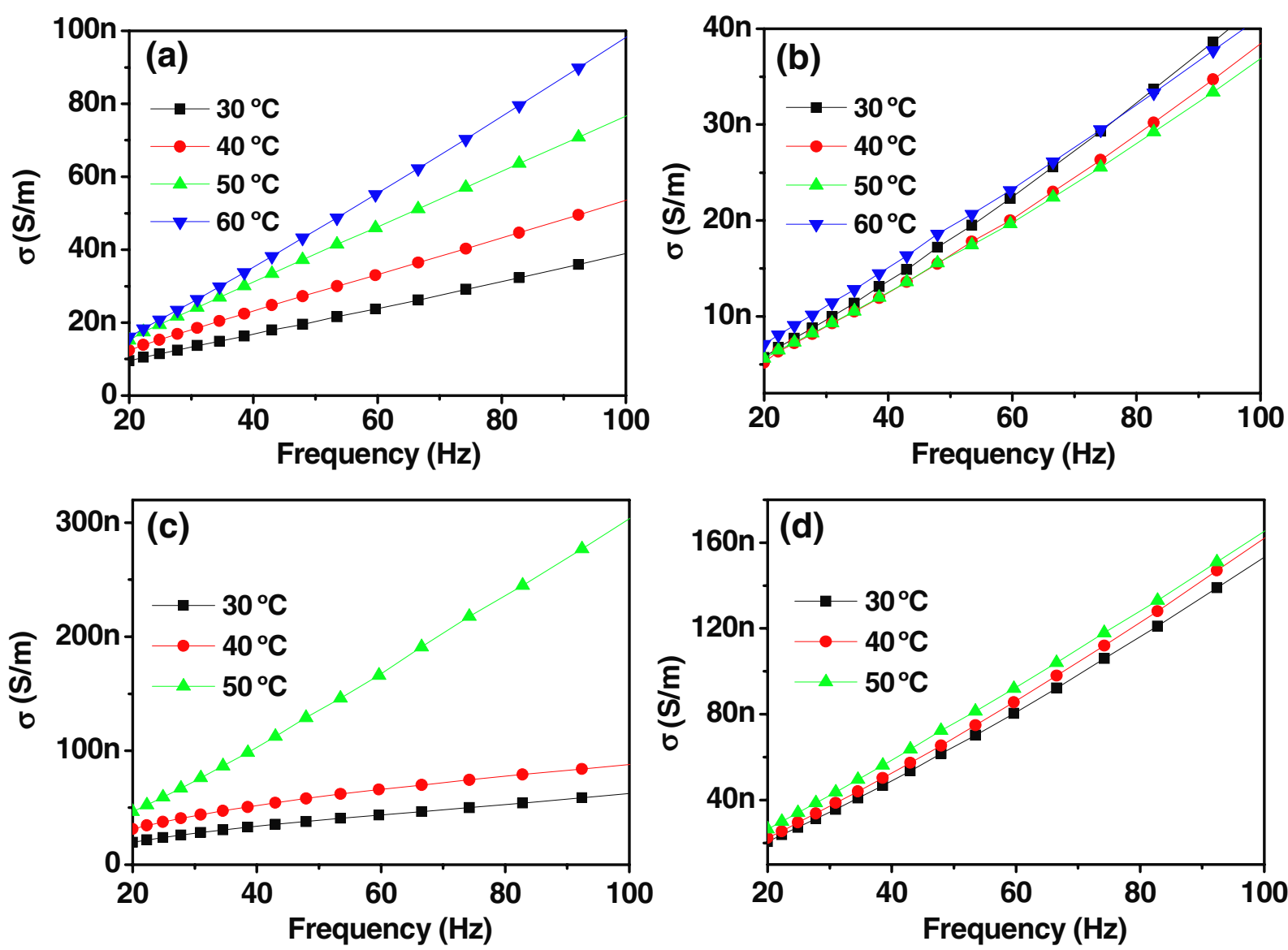

Figure 7. Behaviour of electrical conductivity $(\sigma)$ with frequency of pure and $1 \mathrm{wt} \%$ AL-NPs of size $20-30 \mathrm{~nm}$ doped KCFLC 7S $(a),(b)$ and $\mathrm{KCFLC} \mathrm{10S}(c),(d)$ material, respectively.

AL-NPs doped KCFLC 10S. The suppression of ionic effects of KCFLC $10 \mathrm{~S}$ material has been confirmed by observing the behaviour of $R$ with frequency at different temperatures (figure 6(b)). The increased value of $R$ in the case of AL-NPs doped KCFLC 10S material clearly indicated the minimization of ionic effects due to their adsorption on the AL-NPs surfaces.

Since electrical resistance is the characteristic of the particular cell whereas the electrical conductivity $(\sigma)$ is the characteristic of the material; we calculated and plotted the electrical conductivity of pure and $1 \mathrm{wt} \%$ AL-NPs doped KCFLC 7S/10S materials (figure 7). It is clear from the figure that the value of $\sigma$ was increased with increase in temperature for both pure and doped materials. In the case of AL-NPs doped KCFLC 7S material (figure 7(b)), the value of $\sigma$ was significantly reduced. For instance, at $50^{\circ} \mathrm{C}$ and $60 \mathrm{~Hz}$ frequency, its value was $46 \mathrm{~S} \mathrm{~m}^{-1}$ for pure KCFLC 7S (figure 7(a)) while for AL-NPs doped KCFLC 7S material it reduced to $19.6 \mathrm{~S} \mathrm{~m}^{-1}$ (figure $7(b)$ ). The same behaviour (decrease in the value of $\sigma$ ) was observed in the case of $1 \mathrm{wt} \%$ AL-NPs doped KCFLC 10S material (figures $7(c)$ and $(d)$ ).

The effect of AL-NPs on the dielectric permittivity $\left(\varepsilon^{\prime}\right)$ has also been observed. Figure 8 shows the variation of $\varepsilon^{\prime}$ of pure and $1 \mathrm{wt} \%$ AL-NPs doped KCFLC 7S material with frequency at different temperatures. It is observed that the value of $\varepsilon^{\prime}$ for AL-NPs doped material was decreased as compared with that of the pure one. The decrease in $\varepsilon^{\prime}$ can be understood by the fact that it is directly related to the net charge stored within a LC cell which acts as a capacitor. The presence of ions in FLC material can contribute largely to $\varepsilon^{\prime}$ in the low-frequency regime resulting in the higher values of $\varepsilon^{\prime}$. The doping of AL-NPs in KCFLC 7S has adsorbed these ionic impurities which in turn resulted in the form of lower values of $\varepsilon^{\prime}$ in the low-frequency regime. Another possible reason may be the lowering of effective polarization due to presence of AL-NPs. It is evident from the behaviour of $\tan \delta$, permittivity and resistance that the doping of AL-NPs has resulted in remarkable suppression of ionic impurities present in KCFLC 7S material. The effect of cell thickness on the ion adsorption capability of AL-NPs has also been analysed. We made the sample cells of different thicknesses and filled them with KCFLC 7S/AL-NPs mixture. We found that the suppression of ionic effects was poor in the case of thin cells $(<3 \mu \mathrm{m})$ whereas they have strongly been reduced in the case of the cells having thickness more than $3 \mu \mathrm{m}$. The dependence on ion adsorption capability of AL-NPs on the cell thickness is not clear at this stage. It needs some further careful investigations.

The reduction of ionic impurities of FLCs by doping AL-NPs did not show degradation over a long time. The experiments were repeated on the same sample cells up to 150 days and ionic effects were not found in the AL-NPs doped KCFLC 7S/10S materials with no degradation in contrast being 

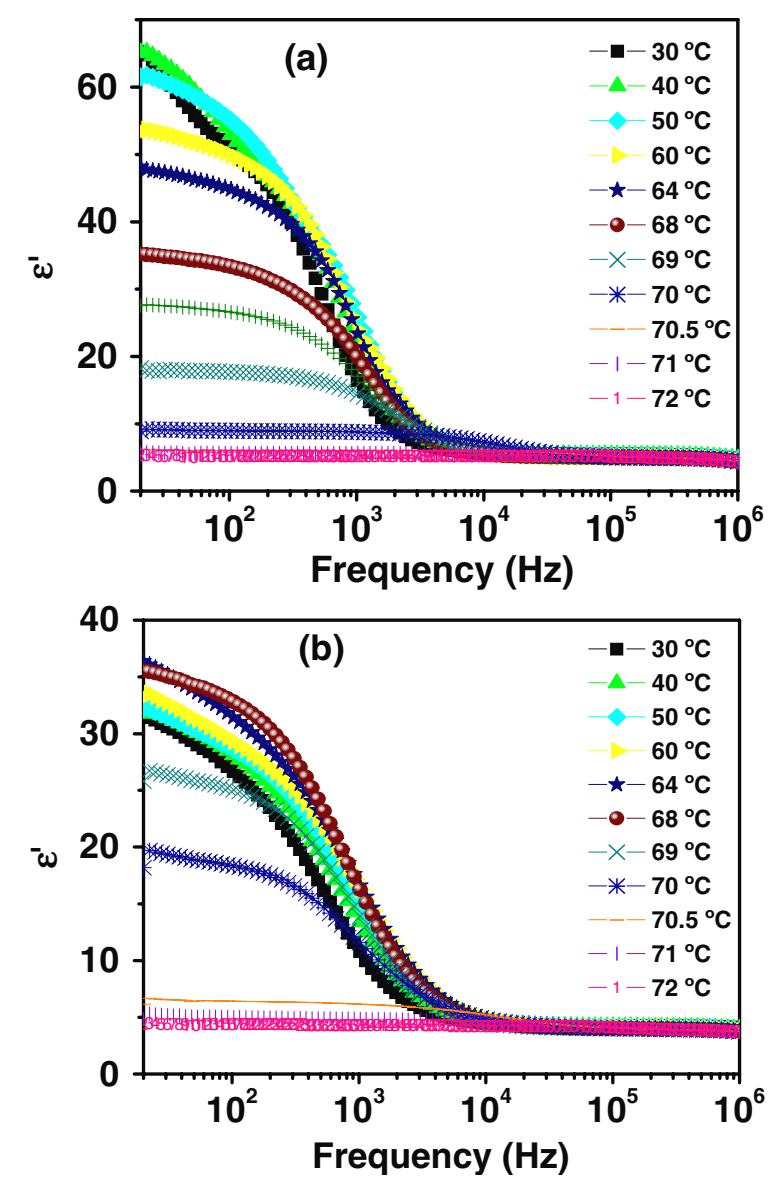

Figure 8. Behaviour of dielectric permittivity $\left(\varepsilon^{\prime}\right)$ of $(a)$ pure and (b) $1 \mathrm{wt} \%$ AL-NPs of size 20-30 nm doped KCFLC 7S material with frequency for homogeneously aligned $4.2 \mu \mathrm{m}$ thick cells.

observed. This proves the efficient trapping of ion pairs by ALNPs for longer duration.

The adsorption of impurity ions of FLCs by AL-NPs could be understood by taking into account the fact that oxides of metal and non-metals possess the capability of removing the undesired ionic constituents from a gas or liquid stream by adsorbent mechanisms. The use of activated alumina is considered to be an economical method for treating water for the removal of a variety of pollutants, gasses and some liquids [22]. Its highly porous structure allows for preferential adsorptive capacity for moisture and contaminants contained in gasses and some liquids. Mccafferty [23] presented an electrode kinetic model for pit initiation on aluminium taking into account the adsorption of chloride ions on the oxide surface and their penetration through the oxide film by means of oxygen vacancies. In the case of nanoparticles, as crystal size becomes smaller, anion/cation vacancies become prevalent and their surface concentration goes up considerably to affect surface energy and intrinsic chemical reactivity is enhanced. AL-NPs fall in the category of insulator metal oxides. There have been numerous studies of their surfaces in an attempt to clarify the type of defect sites that can exist. The most common defects are coordinatively unsaturated ions due to the presence of planes, edges, corners, anion/cation vacancies and electron excess centres [24]. Therefore, polar species and ions strongly chemisorb on their surfaces. Adsorption of several ions, trace metals [25], potential-determining $\mathrm{H}^{+}$and $\mathrm{OH}^{-}$ions [26] has been studied by researchers. Nanoalumina is efficiently being used for removal of ions due to the greater active sites and large surface area. Afkhami et al observed the simultaneous removal of heavy-metal ions in wastewater samples using nanoalumina modified with 2, 4-dinitrophenylhydrazine [27]. Saxena et al [28] studied the adsorption of sulfur mustard on AL-NPs in pure form as well as with impregnants. A recent study on the adsorption capacity of AL-NPs demonstrated how nickel (Ni) ions can be effectively removed from its aqueous solutions [29]. Thermodynamic parameter calculations such as negative values of free energy indicated the feasibility of adsorption of $\mathrm{Ni}$ (II) on nanoalumina. However, Jayathilaka et al reported the enhancement in the conductivity in polymer system ((PEO) ${ }_{9}$ LiTFSI) by doping AL-NPs (10 wt \%), which was attributed to the creation of additional sites and the interactions between the filler surface groups and the ionic species [30]. This study describes the removal of ionic impurities in FLC/AL-NPs system by means of adsorption phenomena, which requires further careful investigations to understand the exact mechanism of adsorption.

The AL-NPs of different sizes and concentrations were doped into different FLC mixtures and their effect on low-frequency ionic contribution has been analysed by means of dielectric relaxation spectroscopy and resistance measurements. We observed that the doping of AL-NPs in FLCs has considerably suppressed the ionic effects of the FLC materials. The presence of the undesired ionic impurities generally causes the degradation of display devices based on LC materials. We found that doping of $1 \mathrm{wt} \%$ AL-NPs of 20-30 nm size range is suitable to effectively suppress the ionic contributions of FLC material. The study will certainly provide an effective tool to remove the undesired ionic impurities present in the FLC mixtures.

\section{Acknowledgments}

The authors sincerely thank Professor R C Budhani, the Director, National Physical Laboratory, for continuous encouragement and interest in this work. The authors (TJ, AK, JG and JP) are grateful to University Grant Commission (UGC) and Council of Scientific and Industrial Research (CSIR), New Delhi, for providing financial assistance.

\section{References}

[1] Prakash J, Choudhary A, Mehta D S and Biradar A M 2009 Phys. Rev. E 80012701

[2] Joshi T, Kumar A, Prakash J and Biradar A M 2010 Appl. Phys. Lett. 96253109

[3] Prakash J, Choudhary A, Kumar A, Mehta D S and Biradar A M 2008 Appl. Phys. Lett. 93112904

[4] Mada H 1990 Japan. J. Appl. Phys. 29 L123

[5] Yang K H, Chieu T C and Osofsky S 1989 Appl. Phys. Lett. 55125

[6] Zou Z, Clark N A and Handschy M A 1991 Ferroelectrics 121147

[7] Barbero G and Durand G 1990 J. Appl. Phys. 672678 
[8] Perlmutter S H, Doroski D and Moddel G 1996 Appl. Phys. Lett. 691182

[9] V A Tsvetkov and O V Tsvetkov 2001 Mol. Cryst. Liq. Cryst. 368625

[10] Chen P-S, Huang C-C, Liu Y-W and Chao C-Y 2007 Appl. Phys. Lett. 90211111

[11] Chen P-S, Huang C-C, Liu Y-W and Chao C-Y 2009 Mol. Cryst. Liq. Cryst. $\mathbf{5 0 7} 202$

[12] Chen W-T, Chen P-S and Chao C-Y 2009 Japan. J. Appl. Phys. $\mathbf{4 8} 015006$

[13] Lee W, Wang C-T and Lin C-H 2010 Displays 31160

[14] Liu H-H and Lee W 2010 Appl. Phys. Lett. 97023510

[15] Onishi H, Johten K, Uemura T, Sakurai T, Mikami N and Yokota T 1995 US Patent No 5403511

[16] Murakami S and Naito H 1997 Japan. J. Appl. Phys. Part 1 362222

[17] Sierakowski M 2002 Mol. Cryst. Liq. Cryst. 375659

[18] Levstik A, Carlsson T, Filipic C, Levstik I and Zeks B 1987 Phys. Rev. A 353527

[19] Hiller S, Beresnev L A, Pikin S A and Haase W 1996 Ferroelectrics 180153
[20] Kundu S K, Suzuki K and Chaudhuri B K 2003 J. Appl. Phys. 942271

[21] Joshi T, Kumar A, Prakash J and Biradar A M 2010 Liq. Cryst. 371433

[22] Moskovitz M L and Kepner B E 1999 US Patent No 5985790

[23] Mccafferty E 1995 Corros. Sci. 37481

[24] Kenneth J Klabunde 2001 Nanoscale Materials in Chemistry (New York: Wiley)

[25] Pavlova V and Sigg L 1988 Water Res. 221571

[26] Sadek H, Helmy A K, Sabet V M and Tadros T F 1970 J. Electroanal. Chem. 27257

[27] Afkhami A, Tehrani M S and Bagheri H 2010 J. Hazardous Mater. 181836

[28] Saxena A, Sharma A, Srivastava A K, Singh B, Gutch P K and Semwal R P 2009 J. Chem. Technol. Biotechnol. 841860

[29] Sharma Y C, Srivastava V, Upadhyay S N and Weng C H 2008 Indust. Eng. Chem. Res. 478095

[30] Jayathilaka P A R D, Dissanayake M A K L, Albinsson I and Mellander B-E 2002 Electrochim. Acta 473257 